\title{
Role of Steroids in Malignant Bowel Obstruction
}

\section{"Corresponding author}

Rakesh Garg, MD, DNB, PGCCHM

Assistant Professor

Department of Onco-Anaesthesiology

Pain and Palliative Care

Dr. BRA-IRCH

All India Institute of Medical Sciences (AllMS), Room No. 139, $1^{\text {st }}$ Floor

Ansari Nagar, New Delhi 110029, India

Tel. +91 9810394950; +919868398335

E-mail: drrgarg@hotmail.com

\section{Volume 2 : Issue 2}

Article Ref. \#: 1000PMHCOJ2116

\section{Article History}

Received: June $20^{\text {th }}, 2016$

Accepted: October $3^{\text {rd }}, 2016$

Published: October $7^{\text {th }}, 2016$

\section{Citation}

Patel A, Garg R. Role of steroids in malignant bowel obstruction. Palliat Med Hosp Care Open J. 2016; 2(2): 30-36. doi: 10.17140/PMHCOJ-2-116

\section{Copyright}

(C2016 Garg R. This is an open access article distributed under the Creative Commons Attribution 4.0 International License (CC BY 4.0), which permits unrestricted use, distribution, and reproduction in any medium, provided the original work is properly cited.

\author{
Anuradha Patel, MD, Rakesh Garg, MD, DNB, PGCCHM ${ }^{*}$ \\ Department of Onco-Anaesthesiology, Pain and Palliative Care, Dr. BRA-IRCH, All India \\ Institute of Medical Sciences (AIIMS), New Delhi, India
}

\section{ABSTRACT}

Various cancers such as ovarian, stomach, colon or pancreas may present with mechanical bowel obstruction. This may be partial or total depending on the pathology and extent of the disease. On presentation, the patient requires appropriate assessment and management as per underlying pathology and assessment findings. Comprehensive medical and surgical management strategies are required to manage malignant bowel obstruction effectively. Steroids have been reported for their beneficial use in malignant bowel obstruction. We review the usefulness of steroids in patients presenting with malignant bowel obstruction.

KEYWORDS: Malignant Bowel Obstruction (MBO); Steroids; Cancer.

ABBREVIATIONS: MBO: Malignant Bowel Obstruction; EC: Enterochromaffin; PEG: Percutaneous Endoscopic Gastrostomy; RCT: Randomized Controlled Trials; NCCN: National Comprehensive Cancer Network; BPCC: Bristol Palliative Care Collaborative.

\section{INTRODUCTION}

Bowel obstruction refers to functional or mechanical obstruction of the intestine which prevents physiological passage of food through the bowel. The bowel obstruction may be partial or complete and depends on the underlying pathology and extent of the disease. If such obstruction occurs due to malignancy, it is referred as malignant bowel obstruction (MBO). It can occur from an intraabdominal malignancy or related to malignancy with peritoneal involvement. The global prevalence of MBO is 3-15\% of cancer patients. ${ }^{1}$ MBO occurs more commonly in women (range: $59-69 \%$ ), ${ }^{1}$ and the age at presentation ranges from $58-65$ years. ${ }^{1,2}$ The time to manifestation of MBO from diagnosis of cancer is around 14 months (range 13-15 months). ${ }^{1}$ The MBO occurs most commonly in the small bowel (61\%) followed by the large bowel (33\%) but may occur in both the small and large bowel simultaneously in $20 \%$ of patients. ${ }^{2} \mathrm{MBO}$ is seen in $20-50 \%$ of patients with ovarian cancer, $6-19 \%$ of stomach cancer patients, $6-13 \%$ of pancreatic cancer patients, and in $3-10 \%$ of patients with urinary bladder cancers. ${ }^{1}$ Also, metastatic peritoneal deposits from breast cancer and melanoma lead to $\mathrm{MBO}$ in around 2-3\% respectively. ${ }^{1}$ The operative mortality $(9-40 \%)$ and complication rates (9-90\%) are very high with overall survival from 3-6 months. ${ }^{2}$ The MBO related to mortality is observed in $15 \%$ of patients receiving palliative care. ${ }^{3}$

\section{PATHOPHYSIOLOGY}

The occurrence of MBO in cancer patients usually depends on the extent of disease and is more frequently seen in advanced cancers. MBO may occur because of extrinsic bowel compression, endoluminal bowel obstruction, intramural bowel infiltration, or extensive mesenteric infiltration. ${ }^{1}$ Intra-luminal bowel tumor occludes the lumen of the bowel leading to obstruction and may also lead to intussusception. Intramural bowel tumor extending to mucosa may impair peristalsis or cause blockage of lumen. ${ }^{2}$ Extramural obstruction occurs due to enlargement of tumor, mesenteric or omental masses or due to malignant adhesions or fibrosis. The neural 
infiltration like that of enteric or celiac plexus by the tumor may lead to severe impairment in peristalsis and consequent obstruction due to dysmotiliy. ${ }^{1,2}$

MBO leads to accumulation of fluid and gases causing bowel distension proximal to occlusive pathology. ${ }^{1,3}$ This causes increased intra-luminal pressure and enhanced peristaltic contractions to overcome the obstruction causing colicky pain. Various inflammatory mediators are released including prostaglandins, vasoactive intestinal polypeptide and nociceptive mediators from the enterochromaffin (EC) cells of the intestine. These mediators lead to colicky pain, edema and hyperemia of bowel. ${ }^{1}$ The obstruction is associated with an increase in intraluminal secretion of sodium, water and chlorides leading to nausea or vomiting. Vomiting leads to water and electrolyte losses. ${ }^{3}$ It affects metabolic and hemodynamic status in such patients. Diagnosis is usually made by reviewing the history, comprehensive physical examination and radiological assessment.

\section{MANAGEMENT}

Various strategies for management of MBO include conservative medical management, surgical intervention or in certain cases combination of both. ${ }^{2,3}$ The management is individualized based on clinical assessment and radiological findings with the understanding of background of underlying pathology. Management of MBO depends on the level of obstruction, disease extent, prior anticancer treatment, stage of cancer, prognosis, age, concurrent illness, presence of ascites, risk of repeat or multiple obstruction and patients health and performance status. The final management decision should include patient and family members in addition to palliative physician, gastroenterologist, interventional radiologist, and medical and surgical oncologist. A holistic multidisciplinary team approach with early involvement of the palliative care team is required to deal with the seriousness of the MBO.

The primary goal of $\mathrm{MBO}$ management is effective symptom control and improving quality of life. ${ }^{4}$ The unmet desire of the patient to eat and enjoy a regular diet adds to psychological distress. At times, these patients may require palliative surgical diversion (diversion colostomy or ileostomy) for symptomatic control, especially in patients with large lesions and multiple areas of obstruction. Endoscopic stent placement and percutaneous endoscopic gastrostomy (PEG) placement are indicated in patients who are having poor physical status for surgery and refractory to medical management. ${ }^{4}$

Medical management is suitable for patients with MBO wherein expected survival is just weeks to days or in those who are poor surgical candidates. ${ }^{5}$ The surgical intervention is not suitable in patients of $\mathrm{MBO}$ associated with carcinomatosis, palpable intraabdominal tumor mass with advanced disease, bowel obstructions at multiple sites, exposure to radiation therapy, and poor functional status. ${ }^{5}$ The main aim of medical or surgical intervention is symptom management including control of nausea, vomiting, pain, maintenance of feeding, and optimization of biochemical abnormalities, thus allowing patients to be manageable at home with improved quality of life. Conservative management includes pharmacotherapy, intravenous fluid for hydration, nasogastric tube for decompression and psychosocial support.

The patient with MBO requires various pharmacological therapies for different purposes. ${ }^{6}$ Opioids like fentanyl and morphine are used for pain management. Doses of opioids should be titrated per patient response, and there is no ceiling dose. Anticholinergics (hyoscine, scopolamine, glycopyrrolate) are used to slow peristaltic contractions..$^{6-8}$ Antisecretory drugs include thesomatostatin analogue (octreotide). ${ }^{2,7}$ Antiemetics used include dopamine antagonists (haloperidol, metoclopramide, chlorpromazine), serotonin antagonist (ondansetron, granisetron) and steroids (dexamethasone)..$^{2,7,8}$ The appropriate and timely medical management of $\mathrm{MBO}$ provides spontaneous resolution in more than one-third of patients. ${ }^{2}$

\section{ROLE OF STEROIDS}

The treatment targets three basic pathophysiologic consequences of MBO i.e. cascade of secretion, distension and bowel hypertonia associated with MBO. ${ }^{6}$ Corticosteroids have been prescribed in palliative therapy since the late 1950 's. They can be given by various routes including intravenous (i.v.), subcutaneous (s.c.), oral and rectal. The commonly used steroids include dexamethasone and methylprednisolone. The bioavailability of oral administration of dexamethasone is $80 \%{ }^{9}$ Anti-inflammatory potency of dexamethasone is 5-10 times as that of methylprednisolone.

Steroids have a central antiemetic, anti-inflammatory, anti-secretory, analgesic and non-specific effect on general wellbeing when administered for MBO. They decrease gut wall edema, peritoneal inflammation and inflammation in proximity to the obstruction. They also decrease excretion of water and salt into the bowel lumen and thus indirectly decrease pain. Steroids decreased European Medicines Agency (EMA) at the site of bowel lumen obstruction due to tumor (extrinsic or intrinsic) and thus are beneficial in relieving MBO. Intestinal transit also improves because of decreased perineural edema and thus has bimodal benefit of both symptomatic relief and reduction in obstruction. ${ }^{10}$ Thus, steroids hasten resolution of MBO and shorten length of hospital stay. Their general effect includes improved appetite, mood and strength as well.

The type of steroid, its dose and dosing schedule have been variously reported in literature. There is absence of recommendations for the specific steroid to be used and the specific dose for MBO. Below is a review of the existing literature for the use of steroids in MBO.

\section{Studies Evaluating the Role of Steroids in MBO}

Hardy et $\mathrm{al}^{11}$ reported a randomized double blind placebo controlled trial in patients with gynecological cancer. He observed 
bowel obstruction resolution in 13 of 35 patients with $4 \mathrm{mg}$ intravenously dexamethasone given every 6 hours (total $16 \mathrm{mg}$ / day) for 5 days. Resolution of MBO occurred in $60 \%$ of patients. Side effects noted were gastrointestinal toxicity and abnormal perianal sensation. However, numbers of patients studied were too small for statistical analysis. ${ }^{11}$

Philip et $\mathrm{al}^{12}$ reported a retrospective study in patients with gynecological cancer with MBO. They concluded that dexamethasone given in a dose of $8 \mathrm{mg}$ /day intravenously or subcutaneously for a minimum of 3 days caused restoration of gastrointestinal function in 5 and symptomatic improvements in 4 patients out of total 13 patients. These effects were sustained for more than one month. The reduction in pain, nausea and vomiting with improved oral intake was observed in $69 \%$ patients in this study. However, absence of control group and very small sample size was limitation of this study. ${ }^{12,13}$

Laval et $\mathrm{al}^{14}$ reported a randomized double-blind prospective study in patients with MBO. In this study, methylprednisolone 40 or $240 \mathrm{mg}$ was administered once a day intravenously over 1 hour for 3 days. They concluded that out of 52 patients, $68 \%$ responded to treatment as compared to $33 \%$ on placebo. Outcomes were not statistically significant and because of the small sample size, no conclusion was made regarding dosage and efficacy. ${ }^{14}$

Laval et $\mathrm{al}^{15}$ recommended use of steroids in peritoneal carcinomatosis with a dose of $1-4 \mathrm{mg} / \mathrm{kg} /$ day methylprednisolone and $0.25-1 \mathrm{mg} / \mathrm{kg} /$ day dexamethasone once a day intravenously or subcutaneously for a short course (5-10 days). They suggested steroids be started at the time of diagnosis and continued if symptoms improve or with resolution of bowel obstruction. Its long-term use is not recommended. ${ }^{15}$

\section{META-ANALYSIS ON USE OF STEROIDS IN MBO}

Feuer et al $^{16}$ reviewed 3 unpublished, randomized, placebo, double-blind controlled trials and 7 published (retrospective and prospective) trials. This meta-analysis reported that 6-16 $\mathrm{mg}$ intravenous dexamethasone per day was found to reduce the symptoms and resolve MBO in advanced gynecological and gastrointestinal cancer. Side effects were extremely low, but there was no survival benefit and the results were not statistically significant. This analysis also reported that six patients were needed to be treated for $\mathrm{MBO}$ with steroids to resolve one episode of bowel obstruction. . $^{16,17}$

A Cochrane review in 2008 analyzed 10 trials including 3 unpublished randomized controlled trials (RCT) and 7 published prospective and retrospective trials accountable for 89 patients. ${ }^{17}$ They concluded that that the dose range 6-16 mg dexamethasone given intravenously or subcutaneously may bring about the resolution of bowel obstruction in $60 \%$ patients. The incidence of side effects is extremely low. However, it does not affect the length of survival. ${ }^{17}$

\section{Studies Evaluating the Role of Steroids in Combination with Other Drugs in MBO}

Mercadante et al ${ }^{18}$ reported a study wherein he administered combination of metoclopramide, octreotide, steroids and amidotrizoate for MBO. The authors concluded these drugs an appropriate combination and doses provided symptom resolution in the palliative care setting. These drugs led to resolution of MBO along with improved intestinal mobility within 1-5 days and maintained bowel patency until death in most of the patients. ${ }^{18}$

Mercadante ${ }^{19}$ studied combination of metoclopramide, octreotide and dexamethasone $12 \mathrm{mg}$ intravenous infusion with amidotrizoate in MBO. He founded that out of 15 patients, 14 had recovery of intestinal transit within 1 to 5 days due to synergistic effect and vomiting resolved within 24 hours. ${ }^{19}$

Laval et $\mathrm{al}^{20}$ observed that relief of MBO occurred with steroids along with antiemetic anticholinergic and analgesic when given for 5 days. They reported that 25 patients (31\%) had symptomatic control without obstruction relief and 25 patients (31\%) had resolution of MBO out of total 80 cases. $^{20}$ The dose of methylprednisolone use was $1-4 \mathrm{mg} / \mathrm{kg} / 24$ hours for $1 \mathrm{hr}$ intravenous infusion in the morning for 5 days or subcutaneously in 2 fractions in 2 different sites.

Murakami $\mathrm{H}$ et $\mathrm{al}^{21}$ studied a combination of octreotide acetate and steroids in 19 patients with MBO. They observed that 13 patients out of 19 showed a marked resolution of the $\mathrm{MBO}$, four patients showed a good response and no response was seen in 2 patients. They also reported that steroids increased the efficacy of octreotide for MBO symptom resolution. ${ }^{21}$

\section{GUIDELINES FOR STEROID USE}

According to National Comprehensive Cancer Network (NCCN) guidelines for palliative care, intravenous dexamethasone is to be given at a dose of $4 \mathrm{mg} 3$ to 4 times a day for MBO. ${ }^{5}$ It should be stopped if no improvement occurs within 3-5 days.

Ahmad $\mathrm{F}^{22}$ established current standards and guidelines for medical management of malignant bowel obstruction. He proposed that steroids are useful in patients with MBO for symptom management. He proposed that in patients with $\mathrm{MBO}$, a 5-day trial of dexamethasone $8 \mathrm{mg}$ once a day or $4 \mathrm{mg}$ twice a day subcutaneously should be considered unless contraindicated. ${ }^{22}$ Though the length of survival is not affected it improves the quality of life by symptom resolution. He also proposed that after administration of steroids for 48 hours, if the symptom resolves then the steroid should be tapered to lowest dose required to symptom control. Steroids should be discontinued if symptom improvement is not seen in 5 days of its administration. ${ }^{22}$

Bristol Palliative Care Collaborative (BPCC) steroid 
guidelines $^{23}$ have recommended 4-8 $\mathrm{mg}$ dose of dexamethasone in $\mathrm{MBO}$.

\section{DISCUSSION}

Steroids are one of the drug classes prescribed for patients requiring palliative care for management of advanced malignancy for various symptom management. ${ }^{24}$ Dexamethasone is relatively inexpensive, easily administered, well tolerated and may be given at home. Since steroids do have associated side effects, their use should be justified, used for minimum period and the minimal dose and its benefits should outweigh the adverse profile. Six mg of dexamethasone is comparable to $32 \mathrm{mg}$ methylprednisolone and $40 \mathrm{mg}$ prednisolone as equipotent doses.

Dexamethasone side-effects at dose range of 6-16 mg are minimal if used short-term, but the risk of gastrointestinal ulceration and immune suppression should be appropriately recognized and managed. Prophylactic gastric protection by a proton pump inhibitor should be advised. Common side effects include oral candidiasis, exacerbation of pre-existing diabetes, hypomania, agitation, hyperkinesia, peptic ulcer and insomnia. Total doses should be divided and given at breakfast and lunchtime to reduce psychotropic effects and insomnia. It is recommended that last dose of steroids be taken before $2 \mathrm{pm}$ to reduce suppression of hypo-pituitary-adrenal axis..$^{25}$ The associated side effects of steroids need to be managed whenever prescribed in palliative care setting including good mouth care for prevention of oral thrush, ulcer prophylaxis and blood sugar control. Concurrent use of NSAIDS or bisphosphonates should be avoided. Since both agents, NSAIDs and steroids lead to gastric ulceration, their combination has been reported to have a 15 times greater risks for peptic ulcer disease as compared to patients who are not using these drugs alone. ${ }^{26}$ The side effects of steroids are well documented and quite common. They should be rapidly tapered if no response is seen in 4-5 days. ${ }^{27}$ Twycross reported that steroids may be started at higher dose and subsequently tapering to effect so as not to miss the response. ${ }^{28}$ Or in other words, steroids, should be used at the lowest effective dose for the shortest period for symptom management in the palliative care setting. ${ }^{26}$ According to clinical response, the dose of steroid should be reduced weekly. Long term side effects include increased susceptibility to infections, impaired wound healing, proximal myopathy, avascular bone necrosis, osteoporosis, excessive weight gain, cushingoid habitus and skin changes. They are contraindicated in active acute infections, psychosis and active peptic ulcer disease. Higher dose of steroids are recommended in those taking anticonvulsants such as phenytoin, carbamazepine, valproate and phenobarbitone as these drugs enhance the metabolism of corticosteroids.

\section{Withdrawal of Steroids}

Steroids should be withdrawn once their optimal effect has been achieved, suddenly or gradually based on patient response.

\section{Abrupt withdrawal may be done in following situations ${ }^{23,29.30}$}

- Dexamethasone dose $<4-6 \mathrm{mg} /$ day

- Dexamethasone $<3$ weeks treatment

- No recent repeated courses of steroids

- No risk of relapse of symptoms

- When adverse effects are not anticipated by abrupt withdrawal

\section{Gradual withdrawal may be done in following situations ${ }^{23,30}$}

- Dexamethasone dose $>6 \mathrm{mg} /$ day

- Dexamethasone $>3$ weeks treatment

- Patient has received second dose in evening

- History of repeated courses of systemic steroids

- Adreno-cortical insufficiency

- Risk of relapse of symptoms

- Patients who are taking a short course within 1 year of stopping long term therapy

For gradual withdrawal, if the dose of dexamethasone is more than $2 \mathrm{mg}$ daily, it should be halved every 3-5 days until reaching $2 \mathrm{mg}$. If dose of dexamethasone used is less than $2 \mathrm{mg} /$ day, dose should be reduced by $0.5-1 \mathrm{mg}$ every 5-7 days. ${ }^{23}$ Initially, the dose of steroids should be reduced rapidly i.e. reducing to half every day so as to reach a physiological requirement i.e. dexamethasone $1-2 \mathrm{mg} / 24 \mathrm{~h}$ or prednisolone $7.5 \mathrm{mg} / 24 \mathrm{~h}$. Subsequently, more gradual reduction is advised. If the symptoms are steroid responsive, it may be continued at a set minimal dose for a maximum of 2-4 weeks. However, the symptom and side effect profile of steroids should be reviewed regularly for early stoppage. If symptom recurs during dose reduction, the dexamethasone dose may be increased back to dose at which symptoms were controlled. ${ }^{30}$

The issues related to steroid withdrawal also include hypo-adrenal crisis-malaise, profound weakness, hypotension, nausea and vomiting, anxiety, terminal restlessness etc. Hence, staged withdrawal with monitoring of these symptoms requires attention and management. Gradual reduction reduces the risk of symptom recurrence and gives time for intrinsic production of steroids to recommence.

\section{CONCLUSION}

$\mathrm{MBO}$ is a seen in palliative care settings commonly in specific types of malignancies. However, there is no definitive standardized evidence-based medical management protocol or guidelines. Various administration routes, optimal doses, duration and type of steroids are yet to be standardized. The existing literature is scarce especially the well conducted prospective randomized studies. The clinical trials reported are having small size, methodological flaws and without statistical significance. Because of intermittent nature of early symptoms associated $\mathrm{MBO}$, it is 

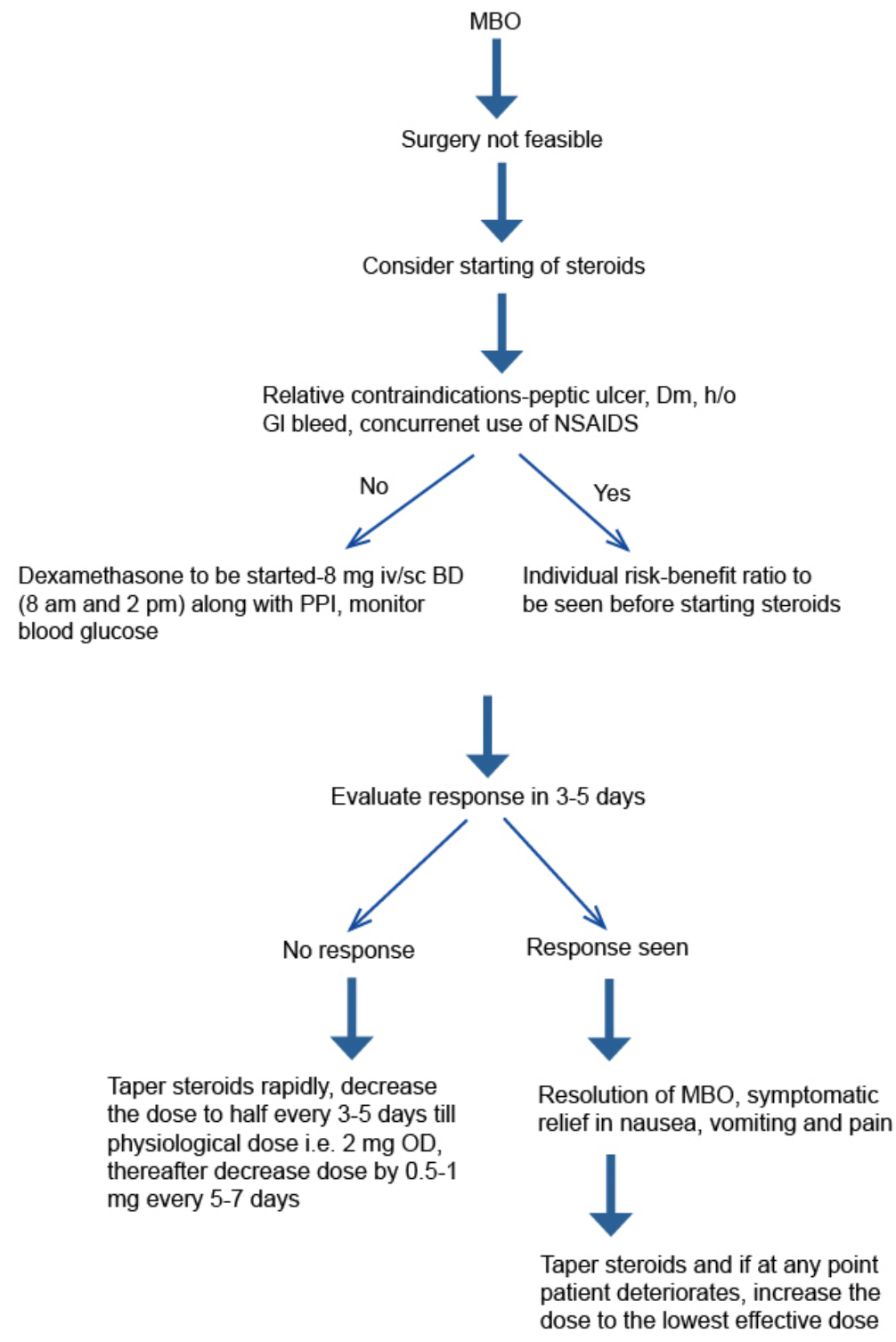

Figure 1: Role of steroids for MBO management.

difficult to attribute resolution of symptoms to steroids. However, the existing literature favors the early use of steroids in patients with MBO. Further, studies and research in large scale is required to statistically prove the efficacy of dexamethasone.

\section{SUGGESTIONS}

Based on published literature, it is suggested to start subcutaneous or intravenous dexamethasone in dose of $8 \mathrm{mg}$ twice a day at 8 am and $2 \mathrm{pm}$ after meals under gastrointestinal prophylaxis for 5 days in patients with MBO with non-feasibility of surgery (Figure 1). Regular assessment and documentation of the response should be done. If no response occurs in 5 days, it should be tapered off quickly. If resolution of bowel obstruction or symptomatic improvement occurs, it should be continued but dose should be tapered to lowest dose required clinically.
General principle of steroid use is-the lowest effective dose for the least possible time. Multimodal treatment of MBO is recommended including anticholinergic, antiemetic, anti-secretory and steroids.

\section{CONFLICTS OF INTEREST}

The authors declare that they have no conflicts of interest.

\section{REFERENCES}

1. Tuca A, Guell E, Losada EM, Codorniu N. Malignant bowel obstruction in advanced cancer patients: Epidemiology, management, and factors influencing spontaneous resolution. Cancer Manag Res. 2012; 4: 159-169. doi: 10.2147/CMAR.S29297 
2. Ripamonti C, Twycross R, Baines M, et al. Clinical-practice recommendations for management of bowel obstruction in patients with end-stage cancer. Support Care Cancer. 2001; 9: 223233.

3. Fainsinger R, Spachynski K, Hanson J, et al. Symptom controlin terminally ill patients with malignant bowel obstruction (MBO). J Pain Symptom Manage. 1994; 9(1): 12-18. doi: 10.1016/0885-3924(94)90141-4

4. Reenan J. Surgery for bowel obstruction in ovarian cancer. AMA J Ethics. 2004; 6: 1-3. Web site. http://journalofethics.amaassn.org/2004/10/cprl1-0410.html. Accessed June 19, 2016.

5. Levy MH, Back A, Baker JN, et al. NCCN (National Comprehensive Cancer Network) clinical practice guidelines in oncology: Palliative care (version 2.2013). J Nati Compr Canc Netw. 2013; 1: 18-19.

6. Ripamonti CI, Easson AM, Gerdes H. Management of malignant bowel obstruction. Eur J Cancer. 2008; 44: 1105-1115.

7. Potluri V, Zhukovsky DS. Recent advances in malignant bowel obstruction: An interface of old and new. Curr Pain Headache Rep. 2003; 7: 270-278. doi: 10.1007/s11916-003-0047-8

8. Tradounsky G. Palliation of gastrointestinal obstruction. Can Fam Physician. 2012; 58: 648-652. Web site. https://www.ncbi. nlm.nih.gov/pmc/articles/PMC3374686/. Accessed June 19, 2016.

9. Duggan DE, Yeh KC, Matalia N, Ditzler CA, McMahon FG. Bioavailability of oral dexamethasone. Clinical Pharmacy and Therapeutics. 1975; 18(2): 205-209. doi: 10.1002/ cpt1975182205

10. MercadanteS, Casuccio A, Mangione S. Medical treatment for inoperable malignant bowel obstruction. J Pain Symptom Manage. 2007; 33(3): 217-223. doi: 10.1016/j.jpainsymman.2006.06.014

11. Hardy J, Ling J, Mansi J, et al. Pitfalls in placebo-controlled trials in palliative care: dexamethasone for the palliation of malignant bowel obstruction. Palliat Med. 1998; 12: 437-442. doi: $10.1191 / 026921698666334766$

12. Philip J, Do T, Lickiss N, et al. Corticosteroids in gastrointestinal obstruction in patients with gynaecological cancer. Int $J$ Gynecol Cancer. 1997; 7: 87.

13. Philip J, Lickiss N, Grant PT, Hacker NF. Corticosteroids in the management of bowel obstruction on a gynecological unit. Gynecol Oncol. 1999; 74: 68-73. doi: 10.1006/gyno.1999.5406

14. Laval G, Girarder J, Lassauniere J, et al. The use of steroids in the management of inoperable intestinal obstruction in ter- minal cancer patients: Do they remove the obstruction? Palliat Med. 2000; 14(1): 3-10. doi: 10.1191/026921600669298725

15. Laval G, Benazech BM, Guirimand F, et al. Recommendations for bowel obstruction with peritoneal carcinomatosis. $J$ Pain Symptom Manage. 2014; 48: 75-91. doi: 10.1016/j.jpainsymman.2013.08.022

16. Feuer D, Broadley KE. Systematic review and meta-analysis of corticosteroids for the resolution of malignant bowel obstruction in advanced gynaecological and gastrointestinal cancers. Ann Oncol. 1999; 10: 1035-1041. doi: 10.1023/A:1008361102808

17. Feuer DJ, Broadley KE. Corticosteroids for the resolution of malignant bowel obstruction in advanced gynaecological and gastrointestinal cancer. Cochrane Database Syst Rev. 2000; CD001219. doi: 10.1002/14651858.CD001219

18. Mercadante S, Avola G, Maddaloni S, Salamone G, Aragona F, Rodolico V. Octreotide prevents the pathological alterations of bowel obstruction in cancer patients. Support Care Cancer. 1996; 4: 393-394. doi: 10.1007/BF01788848

19. Mercadante S, Ferrera P, Villari P, et al. Aggressive pharmacological treatment for reversing malignant bowel obstruction. J Pain Symptom Manage. 2004; 28(4): 412-416. doi: 10.1016/j. jpainsymman.2004.01.007

20. Laval G, Arvieux C, Stefani L, et al. Protocol for the treatment of malignant inoperable bowel obstruction: A prospective study of 80 cases at Grenoble University Hospital Center. J Pain Symptom Manage. 2006; 31: 502-512. doi: 10.1016/j.jpainsymman.2005.10.009

21. Murakami H, Matsumoto H, Nakamura M, Hirai T, Yamaguchi Y. Octreotide acetate steroid combination therapy for malignant gastrointestinal obstruction. Anri Cancer Research. 2013; 33: 5557-5560. Web site. http://ar.iiarjournals.org/content/33/12/5557.long. Accessed June 19, 2016.

22. Ahmad F, Jeffries C, Longford E, et al. Guidelines for the medical management of malignant bowel obstruction. Cheshire and Merseyside palliative and end of life care strategic clinical network group guidelines. NHS. 2015. Web site. Www. cmscnsenate.nhs.uk. Accessed July 1, 2016.

23. Bhatia R, Reid C. BPCC (Bristol Palliative Care Collaborative) steroid guidelines. Palliative care guidelines for the use of steroids in patients with cancer. $P$ T. 2016; 18: 205-209. Web site. Www.stpetershospice.org.uk. Accessed June 19, 2016.

24. Shih A, Jackson KC. Role of corticosteroids in palliative care. J Pain Palliat Care Pharmacother. 2007; 21: 69-76. doi: 10.1080/J354v21n04_14

25. The Bone and Tooth Society, National Osteoporosis So- 
ciety. Glucocorticoid-induced osteoporosis: A concise guide for prevention and treatment. London, UK: Royal College of Physicians; 2002. Web site. http://www.dbh.nhs.uk/Library/ Pharmacy_Medicines_Management/Formulary/Formulary_S6/ Glucocortoid-induced\%20Osteoporosis.pdf. Accessed June 10, 2016.

26. CSM/MCA (Committee on safety of medicines and medicines control agency). Withdrawal of systemic corticosteroids. Current Problems in Pharmacovigilance. 1998; 24: 5A.

27. Piper JM, Ray WA, Daugherty JR, Griffin MR. Corticosteroid use and peptic ulcer disease: Role of non-steroidal anti-inflammatory drugs. Ann Intern Med. 1991; 114: 735-740. Web site. http://annals.org/aim/article/1077526/corticosteroid-use-peptic -ulcer-disease-role-nonsteroidal-anti-inflammatory-drugs. Accessed June 19, 2016.

28. Twycross R. Corticosteroids in advanced cancer. BMJ. 1992; 305: 969-970. Web site. https://www.ncbi.nlm.nih.gov/pmc/articles/PMC1884052/. Accessed June 19, 2016.

29. Landrum LM, Blank S, Chen L, et al. Comprehensive care in gynecologic oncology: The importance of palliative care. Gynecologic Oncology. 2015; 137: 193-202. doi: 10.1016/j. ygyno.2015.02.026

30. Fletcher A, Murray A. Guidelines for use of steroids in cancer patients: Wigan and Leigh Hospice. Web site. www.researchgate.net. Accessed June 10, 2016. 